\title{
Funcionamento Diferencial de Itens para Avaliar a Agressividade de Universitários
}

\section{Differential Items Functioning to Assess Aggressiveness in College Students}

\author{
Fermino Fernandes Sisto ${ }^{a^{*}}$, Daniel Bartholomeu ${ }^{b}$, Acácia Aparecida Angeli dos Santos ${ }^{a}$, \\ Fabián Javier Marín Rueda ${ }^{a} \&$ Adriana Cristina Boulhoça Suehiro ${ }^{c}$ \\ ${ }^{a}$ Universidade São Francisco, Itatiba, Brasil, ${ }^{b}$ Universidade Unianhaguera e Unisal, Americana, Brasil \\ \& ${ }^{c}$ Universidade Federal do Recôncavo da Bahia, Cruz das Almas, Brasil
}

\begin{abstract}
Resumo
Nesta pesquisa buscou-se evidência de validade de construto relacionada ao funcionamento dos itens para diferenciar sexos em um instrumento de agressividade. Participaram 445 universitários, de ambos os sexos, dos cursos de Engenharia, Computação e Psicologia. A escala de agressividade composta por 81 itens foi aplicada coletivamente, em sala de aula, nos estudantes que consentiram em participar do estudo. Os itens do instrumento foram analisados por meio do modelo Rasch. Vinte e oito itens apresentaram funcionamento diferencial, sendo 15 condutas mais características de pessoas do sexo feminino e outras 13 mais características do masculino. Os índices de precisão foram de 0,99 para os itens e 0,86 para as pessoas. Conclui-se que a agressividade pode ser medida separadamente em razão do sexo.

Palavras-chave: Comportamentos agressivos; ensino superior; funcionamento diferencial dos itens.
\end{abstract}

\begin{abstract}
In this research evidences of construct validity were searched analyzing the differential functioning items related to aggressiveness. The participants were 445 college students of both genders, attending the courses of Engineering, Computing and Psychology. The scale of aggressiveness composed by 81 items was collectively applied, in the classroom, to the students who consented to participate in the study. The items of the instrument were studied by means of the Rasch model. Twenty-eight items presented differential functioning item, 15 were characterized as typical for females and 13 for males. The reliability coefficients were 0.99 to the items and 0.86 to the persons. It was concluded that the aggressiveness can be measured separately on the basis of gender. Keywords: Agressive behaviors; higher education; differential items functioning.
\end{abstract}

A agressividade tem sido estudada por vários autores em uma diversidade de contextos e enfoques teóricos. Embora as pessoas apresentem uma grande variedade em seus níveis de agressividade, a agressão extrema tem sido incluída no domínio da psicopatologia, seja por si mesma ou associada a outras patologias (Huesmann, Eron, Lefkowitz, \& Walder, 1984; Jozef, Greenhalgh, Leite, \& Ferreira, 2000; Kolko, Kazdin, \& Meyer, 1985).

Comumente confunde-se o termo agressividade com violência, sendo que inúmeras pesquisas que versam sobre a agressividade evidenciam essa indiferenciação entre os termos, chegando, por vezes, a utilizá-los para indicar um mesmo fenômeno (Moreira, 1982; Sisto, 2005; entre outras). Há também autores que fazem uso do termo agressividade para designar comportamentos hostis ou destrutivos (Aquino, 1996; Maldonado \& Williams, 2005; por exemplo).

* Endereço para correspondência: Universidade São Francisco, Faculdade de Psicologia, Rua Alexandre Rodrigues Barbosa, 45, Itatiba, SP, 13251-900. E-mails: fermino.sisto@gmail.com, d_bartholomeu@yahoo.com.br, acacia.santos@saofrancisco.edu.br, marinfabian@yahoo.com.bre dricbs@yahoo.com.br
À parte dessa dificuldade de diferenciação de termos, as condutas agressivas podem ser definidas como ações intencionais que machuquem ou irritem alguém, física ou emocionalmente, sendo que o maior problema nessa definição é mensurar o componente de intencionalidade (Olweus, 1991, 1993; Sisto, 2003). Uma solução que se pode adotar consiste em inferir essa intencionalidade pela freqüência dos comportamentos (Gomide, 2000; Sisto, 2003). Faz-se importante ressaltar que foi com base nessas definições que foram selecionadas as pesquisas descritas neste estudo.

Uma gama bastante grande da pesquisa sobre agressividade e variáveis relacionadas pode ser encontrada na literatura. Pode-se citar como exemplo o estudo de Sisto (2003), que buscou investigar até que ponto as crianças rejeitadas para estudar percebiam-se como agressivas. Mil duzentos e oitenta e um estudantes foram avaliados com o Teste Sociométrico e a Escala de Agressividade para Crianças e Jovens (Sisto \& Bazi, 2000), desenvolvida para mensurar a agressividade nas situações familiar, escolar e geral. $\mathrm{O}$ autor observou um maior nível de aceitação entre os colegas para as meninas do que para os meninos, além do que as crianças mais velhas foram caracterizadas como 
mais agressivas. Os coeficientes de correlação entre os instrumentos foram baixos, sugerindo que nem todas as crianças agressivas foram rejeitadas. Ao lado disso, a análise de grupos extremos da aceitação-rejeição indicou que os estudantes mais aceitos relataram menos condutas agressivas em situação escolar e geral em comparação aos mais rejeitados.

Identificam-se, ainda na literatura, resultados bastante variados quanto a diferenças de sexo em manifestações agressivas, existindo indicativos de que elas dependem do tipo de agressão. Nesse sentido, aparentemente, as agressões físicas são mais características do sexo masculino em comparação à agressão verbal; enquanto que no caso das pessoas do sexo feminino, têm-se demonstrado um maior uso de estratégias indiretas de agressão (Archer, 1991; Cairns, Cairns, Neckerman, Ferguson, \& Gariepy, 1989; Sisto, 2005; Sisto, Bartholomeu, Rueda, \& Granado, 2005).

No estudo de MacIntyre e Cantrell (1995), os autores examinaram as relações entre o tipo de punição recebida durante a infância e atitudes dos adultos sobre uma variedade de situações violentas e agressivas. Partiu-se da premissa de que homens relatariam uma maior aprovação da violência e da agressão que mulheres, como forma de punição usada para discipliná-los. Esta hipótese foi levantada com base em estudos anteriores que sugerem que os homens seriam mais agressivos que as mulheres. Os resultados indicaram diferença significativa entre os sexos para violência e agressão, sendo que os homens obtiveram maiores médias nessas medidas. Não foram encontradas diferenças para o tipo de punição durante a infância. Os homens concordaram mais freqüentemente com a punição física desde que com uma explicação e as mulheres o fizeram desde que punidas durante discussões.

Em algumas pesquisas foi analisada a agressividade relacionada à intoxicação alcoólica e ao sexo. Sob essa perspectiva, Wells e Graham (2003) verificaram que o dano esteve associado significativamente com o nível de intoxicação alcoólica percebido. Além disso, o sexo dos participantes e a hora do dia também se associaram ao envolvimento com álcool na agressão. Mais especificamente, os resultados indicaram que o envolvimento do álcool na agressão tendeu a ser maior em homens com idades de até 30 anos e ocorreu após a meia noite em bares e outros eventos sociais em fins de semana.

As diferenças de sexo quanto aos efeitos do álcool na severidade percebida da agressão foram exploradas por Graham, Plant e Plant (2004). Os participantes do estudo foram questionados sobre seus hábitos de beber e sobre o incidente mais grave de agressão física vivenciado pelo e com o parceiro(a) ou esposo(a), durante os últimos dois anos. Aproximadamente $20 \%$ das pessoas relataram agressão pelo parceiro e em 35 a $40 \%$ dos incidentes houve ingestão de álcool por um ou ambos. O uso do álcool na situação agressiva foi associado com um aumento na gravidade da agressividade, raiva e medo, especialmente por agressores homens sobre mulheres.

Por sua vez, Fillmore e Weafer (2004) compararam homens e mulheres adultos no grau em que uma dose de álcool moderada prejudicaria o controle inibitório. Os sujeitos relataram beber socialmente e na situação experimental receberam uma dose moderada de álcool $(0,65 \mathrm{~g} /$ $\mathrm{kg}$ ) e um placebo, em dois diferentes momentos. Sua tarefa era realizar uma atividade na qual se mensurava a habilidade de inibir e executar respostas comportamentais. Os homens mostraram maiores danos no controle inibitório sob a condição de álcool do que as mulheres, e relataram maiores níveis de excitação, enquanto as mulheres evidenciaram um maior efeito de sedação. Os autores observaram ainda que os comportamentos agressivos dos homens foram mais pronunciados nessas condições em comparação com as mulheres.

A possibilidade de identificar traços específicos de personalidade, tal como comportamentos anti-sociais em relação à criminalidade e abuso de álcool, em duas culturas diferentes, assim como investigar se essas características se desenvolveriam na fase adulta foram as preocupações de Pulkkinen, Virtanen, Klinteberg e Magnusson (2000). Dentre os resultados, observou-se que os criminosos homens que apresentaram problemas com álcool tiveram escores significativamente elevados em traços de personalidade relativos à psicopatia em comparação a outros subgrupos. Os indicadores foram alta impulsividade, tensão muscular e baixa socialização. $O$ grupo de homens criminosos também apresentou altas pontuações na agressividade relatada pelos professores na infância em detrimento do grupo não criminoso. $\mathrm{O}$ grupo feminino que apresentou atividade criminosa foi pequeno e não diferiu significativamente das não criminosas nos traços de personalidade estudados. As mulheres criminosas finlandesas, entretanto, mostraram menos indicações de baixa sociabilidade, de alta agressividade e de problemas na infância em comparação ao grupo de não criminosas suecas.

Os riscos de agressão sexual de homens na universidade foram objetos de estudo de Carr e VanDeusen (2004). Dentre os fatores de risco foram incluídos padrões de abuso de substâncias, consumo de pornografia, atitudes negativas em relação ao sexo e experiências de abuso sexual na infância. A análise de regressão indicou que o uso de pornografia e abuso de álcool foram preditores significativos do envolvimento em violência sexual. Muitos homens ainda relataram fazer uso de coerção sexual relacionada ao uso de álcool e demonstraram atitudes e crenças relacionadas a estupro. $\mathrm{O}$ abuso sexual infantil não foi um preditor da agressão sexual quando adulto.

Em estudo brasileiro, Sisto et al. (2005) procuraram diferenciar manifestações agressivas em razão do sexo de estudantes universitários. Alguns dos resultados encontrados sugeriram que os homens tenderam a importunar mais as outras pessoas, destruir coisas que não lhes pertenciam, enganar e violar regras, desrespeitando a segurança própria e alheia. Também culparam vítimas por coisas que fizeram, praticaram coação para obter favorecimentos, além de não demonstrarem arrependimento pelos seus atos e agredirem pessoas e animais, entre outras manifestações. Por sua vez, manifestações como ser manipulador, irritadiço, usar de ataques de birra para alcançarem ob- 
jetivos, brigar excessivamente, humor deprimido e baixa tolerância a frustrações caracterizariam as mulheres. Nesse sentido, os autores concluíram que certas condutas seriam peculiares a indivíduos mais agressivos de um sexo ou de outro, apontando que novas pesquisas sobre o tema deveriam considerar que as manifestações da agressividade seriam diferentes em razão desses grupos.

Nas publicações são encontrados também outros tipos de estudos em relação à agressividade e sexo. Por exemplo, na pesquisa de Meijer (1991), homens e mulheres adultos foram requisitados para avaliar 16 diferentes expressões de movimentos agressivos, gravados em vídeo, em uma escala de quatro pontos. Cada movimento já apresentava uma classificação em quatro níveis de intensidade, obtida com base em um estudo prévio. Os sujeitos foram instruídos a olhar para o corpo em movimento e não para o rosto e ambas as avaliações deveriam ser feitas imediatamente após a apresentação de cada movimento. Em relação ao sexo, os homens pontuaram mais alto que as mulheres nas agressões em movimentos, classificadas em um nível alto ou moderado de agressão, e forneceram avaliações mais baixas que elas para movimentos em um nível menor de intensidade.

Analisando a literatura pertinente à agressividade, percebeu-se que as diferenças de sexo em comportamentos agressivos estavam presentes, sugerindo que essa variável manifesta-se de formas diferentes para homens e mulheres. Com base nessa informação, pode-se pensar que certas condutas agressivas poderiam ser características de um ou outro sexo, conforme apontam os estudos de Archer (1991), Cairns et al. (1989), Sisto (2005) e Sisto et al. (2005). Tradicionalmente as análises sobre as diferenças entre os sexos são realizadas com base em médias. Não se encontrou pesquisas que estudassem se essas diferenças seriam devidas às reais particularidades de cada grupo ou se os itens do instrumento estariam facilitando a conduta agressiva para o grupo masculino, em detrimento do feminino, ou vice-versa. A suposição subjacente é que a pontuação obtida num instrumento qualquer ocorre não apenas em função da intensidade da manifestação de um construto nos sujeitos. Assim, características irrelevantes, como pertencer a grupos culturais diferentes, por exemplo, podem interagir com o construto produzindo um resultado que reflete o traço latente, mas também outras variáveis indesejáveis (Sisto, 2006; Sisto, Fernandes, \& Fernandes, 2006; Sisto, Rueda, \& Bartholomeu, 2006).

A relevância desse tipo de investigação reside na possibilidade de se utilizar testes que subestimem ou superestimem as diferenças entre certos grupos em função de características irrelevantes para o construto que se está aferindo. Além disso, a importância da identificação de itens com funcionamento diferencial $(D I F)$ na construção de instrumentos se dá na medida em que possibilita buscar as causas dessas diferenças; evitar o uso de itens em um grupo para o qual eles nitidamente não estão adequados; e controlar fatores responsáveis para evitar a construção de itens com os mesmos problemas (Andriola, 2001; Hambleton, 1989; Jensen, 1980; Sisto, 2006; Sisto et al., 2006).
Em razão disso, como um primeiro estudo para a construção de uma escala para mensurar a agressividade em adultos jovens e adultos, optou-se por fazer um estudo sobre os itens com vistas a verificar se o funcionamento dos itens se diferenciava. Trata-se, portanto, de um estudo cujo objetivo é buscar evidência de validade de construto dos itens, com base na análise interna do instrumento, já que ela informa o grau que um item estaria mensurando um traço determinado, possibilitando a identificação de quais deles estariam enviesados, favorecendo um ou outro grupo (American Psychological Association [APA], American Educational Research Association [AERA], \& National Council on Measurement in Education [NCME], 1999).

\section{Método}

\section{Participantes}

Participaram desta pesquisa 445 estudantes universitários dos cursos de Engenharia (31\%), Computação (17\%) e Psicologia (52\%) de uma universidade particular de uma cidade do interior do estado de São Paulo. Suas idades variaram de 18 a 65 anos com média de 25 anos $(D P=7,13)$, sendo que 57,2\% deles tinham até 23 anos. Quanto ao sexo, $57,3 \%$ eram mulheres.

\section{Instrumento e Procedimento}

Com base nas descrições de transtornos de conduta do CID-10 (Organização Mundial de Saúde [OMS], 1993) e do DSM-IV (APA, 1995) foram selecionados os comportamentos agressivos mais relevantes para composição dos itens da escala. Esses comportamentos foram destruir algo que não lhe pertence, importunar os outros, roubar, praticar contravenção, enganar, manipular, mentir, usar nomes falsos, violação de regras, ludibriar, fingir, desrespeitar a segurança própria, desrespeitar a segurança alheia, demonstrar indiferença pela conseqüência de seus atos, culpar as vítimas, atos de coação para obter favorecimento, não se arrepender, crueldade/agressão, minimizar conseqüências danosas de suas ações, incapacidade de tolerar o tédio, baixa tolerância à frustração, auto-suficiência, fazer fofoca, irresponsabilidade persistente, ser provocativo e desafiador, usar termos técnicos para impressionar os outros, humor deprimido, manter relação sexual com menor de idade, fugir, violar regras, brigas excessivas, ataques de birra, irritabilidade persistente, impulsividade, não possui simpatia, uso de substância danosa. A composição dos itens da escala foi feita por sete mestrandos e um aluno de graduação de psicologia.

Inicialmente foram constituídos 151 itens em forma de frases assertivas, os quais foram submetidos a uma análise do conteúdo por cinco juízes. Somente os itens que obtiveram um índice de pelo menos $80 \%$ de concordância foram mantidos na escala. Com base nessa análise, foram excluídos os que apresentavam conteúdos comuns e falta de concordância entre os examinadores, bem como modificados outros que apresentaram problemas na sua compreensão. Assim, restaram 81 itens que foram organizados em escala 
likert de três pontos (nunca, às vezes e sempre). Alguns exemplos de itens da escala são: Altero o tom de voz quando não sou atendido ou entendido, Durante uma conversa não deixo espaço para o outro se colocar, Falo dos outros pelas costas, Informo a meus pais onde realmente vou quando saio, Quando não gosto de alguém sinto vontade de esconder a verdade para prejudicá-lo.

A escala foi aplicada coletivamente, em sala de aula, após a aprovação do projeto pelo comitê de ética e a assinatura do termo de consentimento. $\mathrm{Na}$ aplicação o pesquisador solicitou que a pessoa assinalasse a freqüência de ocorrência dos comportamentos descritos nos itens, sendo que a pontuação mínima que se pôde alcançar foi 81 e a máxima 243.

\section{Análise de Dados}

O modelo de análise de itens sugerido por Rasch (1960) supõe que uma pessoa ao responder a um item expressa certa habilidade ou uma quantidade de um construto. Sendo assim, esse método possibilita o cálculo da probabilidade de que indivíduos num certo nível de habilidade acertem um determinado item com um dado nível de dificuldade; ou que pessoas com níveis mais altos em agressividade, por exemplo, indiquem uma intensidade maior de agressividade em uma série de itens.
A análise de DIF foi feita pelo programa Winsteps. Nesse procedimento são subtraídas as estimativas da intensidade do construto das pessoas dos itens. Em decorrência, supõe-se que a intensidade de cada item deve ser estatisticamente equivalente para os grupos estudados, no caso desta pesquisa o sexo dos sujeitos (Sisto, Noronha, \& Santos, 2005).

Nessa análise é calculado o valor de DIF que é acrescido (positiva ou negativamente) ao item e estimada a significância da diferença por meio da prova $t$ de Student. Utilizouse o critério de Draba (1977) que considera o patamar de 2,4 um bom ponto para indicar a significância estatística ao se analisar um instrumento com mais de 20 itens, pois no presente estudo o número de itens foi de 81 .

\section{Resultados}

Os dados dos itens de agressividade foram submetidos ao modelo de Rasch para verificar sua adequação a uma escala. A precisão do instrumento por esse modelo foi de 0,99 para os itens e 0,86 para as pessoas, indicando uma boa precisão em ambos os casos. O erro de medida médio para as pessoas foi de $0,23(D P=0,04)$ e dos itens foi de $0,12(D P=0,07)$. A Tabela 1 apresenta mais informações.

Tabela 1

Parâmetros de Ajuste de Itens e das Pessoas (N=445)

\begin{tabular}{lccccccccc}
\hline Parâmetros & \multicolumn{3}{c}{ Itens } & \multicolumn{4}{c}{ Pessoas } \\
\cline { 2 - 10 } & Dificuldade & Infit & Outfit & Erro & Habilidade & Infit & Outfit & Erro \\
\hline Média & 0,00 & 1,07 & 0,95 & 0,12 & $-1,87$ & 1,01 & 0,95 & 0,23 \\
DP & 1,25 & 0,17 & 0,21 & 0,07 & 0,64 & 0,29 & 0,53 & 0,04 \\
Máximo & 3,29 & 1,60 & 1,62 & 0,45 & 1,18 & 2,20 & 4,50 & 0,51 \\
Mínimo & $-2,19$ & 0,73 & 0,61 & 0,07 & $-4,09$ & 0,33 & 0,34 & 0,18 \\
\hline
\end{tabular}

Em relação aos itens, a média do infit foi 1,07 ( $D P=0,17)$, indicativo de que, no geral, a maior parte deles foi respondida dentro do padrão esperado $(1,00)$ conforme o modelo de Rasch (Bond \& Fox, 2001). Analisando esses dados mais detidamente, verificou-se que os valores de infit estiveram no intervalo de 1,60 e 0,73 , sugerindo que nem todos os itens enquadraram-se no parâmetro $0,70-1,30$, característico de bom ajuste. Estudando mais detalhadamente os itens com desajustes observou-se que seis apresentaram valores de infit superiores a 1,30, e um caso foi superior a 1,50, valor considerado por Linacre (2002) como o limite máximo para se aceitar um item. Todavia, de modo geral, a variabilidade de infit esteve dentro dos níveis esperados.

Por sua vez, a média de outfit encontrada foi de 0,95 $(D P=0,21)$, o que aponta para uma boa adequação dos itens ao modelo de Rasch. Entretanto, a variação de outfit esteve no intervalo de 0,61 a 1,62, indicando que houve itens fora do intervalo esperado. Mais especificamente, ficou evidenciado que dois itens apresentaram valores acima de 1,50 e quatro abaixo de 0,70 . Vale destacar que apenas um item apresentou desajuste em ambas as medidas com índice acima de 1,50. Dessa forma, 18,5\% dos itens tiveram acertos não esperados em razão da medida de intensidade do construto dos sujeitos; em outros termos, o padrão de resposta foi variado em razão do nível de agressividade das pessoas. Desses, 1,2\% apresentou discrepâncias preocupantes.

No que se refere às pessoas, evidenciou-se que a maioria apresentou o padrão esperado $(1,00)$ em ambos os parâmetros de ajuste, uma vez que a média de infit foi de 1,01 $(D P=0,29)$ e a de outfit de $0,95(D P=0,53)$. Observou-se que o intervalo de variação do outfit $(0,34-4,50)$ foi maior que o de infit $(0,33-2,20)$, sendo que 36 sujeitos $(8 \%)$ evidenciaram índices em ambas as medidas acima de 1,30 e deles $14(3,1 \%)$ forneceram índices acima de 1,50 e outros $28(6,2 \%)$ abaixo de 0,70. Essa quantidade de 
Tabela 2

Mapa de Pessoas e Itens $(N=445)$

4

$$
<\text { mais }>\mid<\text { raro }>
$$

$+$

I

A32

3

0

1

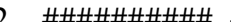

HA\#+

\#\#\#\#\#\# |

.\#\#\#\# SIT

.\#\#\#\#\# I

.\#\# |

$-3$

.\#\# +

.\# $\mathrm{T} \mid$

A31

A28 A46

+ A29 A4

A35 A80

A11 A50

I

IS A17 A45 A63 A79

. I A12

+ A26 A30 A78

A19 A42

A10 A39

A33 A9

A15 A57 A68

I A1 A21 A66

+M A16 A43 A6 A64

$\begin{array}{llll}\text { A22 } & \text { A40 A47 A58 }\end{array}$

$\begin{array}{lllll}.1 & \text { A13 A37 A48 A7 }\end{array}$

. I A20 A61 A72

.\# $\mathrm{Tl} \quad \mathrm{A} 5 \quad \mathrm{~A} 52$

\#\#\# I A51 A73

\#\#\#\# + A14 A18

\#\#\#\# SI $\quad$ A34 A54

\#\#\#\# IS A24 A49

.\#\#\#\#\#\# | A2 A25

$\begin{array}{ll}\text { A2 } & \text { A25 } \\ \text { A53 } & \text { A69 }\end{array}$

A23

A27 A36 A38

A44

A74

A56 A62

A3 A41 A60
A77

A8

A71 A75

A55 A70 desajustes pode, de fato, ser considerada baixa, ainda mais ao se levar em conta o fato de se tratar de uma escala em construção.

Outro aspecto analisado no presente estudo refere-se à análise da variação da dificuldade dos itens da escala. A variação de dificuldade dos itens foi de $-2,19$ a 3,29. Somente um item (A32) apresentou o valor máximo de dificuldade. Aproximadamente metade dos itens apresentou dificuldade entre 1 e -1 logit, intervalo que avalia as pessoas com maior precisão e menos erros. Sua média foi $0,00(D P=1,25)$, indicativo de que os itens evidenciaram dificuldade média para os sujeitos, embora sua dispersão tenha sido grande. Maiores detalhes constam da Tabela 2, na qual a letra $M$ situada ao lado da divisória das informações das pessoas e dos itens informa a posição da média para um e outro.

Os universitários apresentaram média de agressividade de 1,87 $(D P=0,64)$, sugerindo que no geral as pessoas estudadas apresentaram poucas condutas agressivas ou condutas não muito intensas. Também se identificou o intervalo de variação de theta de $-4,09$ a 1,18 , bastante superior ao dos itens, nos quais a maior parte das pessoas apresentou índices entre -1 e -3 logit. Na realidade, a ocorrência da maior parte dos itens nos participantes estudados foi baixa.

Em relação ao funcionamento diferencial dos itens $(D I F)$, a Tabela 3 sumariza os resultados encontrados na análise. Dos 81 itens, 28 (34,57\%) apresentaram resultados dentro dos critérios indicativos de presença de $D I F$. Esse resultado indica que certos itens (condutas agressivas) são mais característicos de um sexo do que de outro. Ao lado disso, $35 \%$ é um valor suficientemente alto e que deve ser levado em consideração ao se construir um instrumento para avaliar a agressividade. Finalmente, também se pôde observar que 15 itens foram condutas muito comuns nas pessoas do sexo feminino e outros 13 nas pessoas do sexo masculino.

Para ilustrar os resultados encontrados realizou-se uma representação gráfica das CCIs do item 3 da Escala, qual seja, "Altero o tom de voz quando não sou atendido ou entendido". Essa informação é apresentada na Figura 1.

$\mathrm{O}$ item 3 apresenta maior probabilidade das pessoas do sexo feminino responderem a ele com maior intensidade, ou seja, o funcionamento diferencial do item é no sentido de favorecer respostas das mulheres, indicando certa predileção a essa conduta em detrimento dos homens. A curva característica do item informa que o intervalo de variação entre os homens e mulheres foi diferente, pois para os homens variou de $-7 \mathrm{a}+4$, enquanto que para as mulheres de $-8 \mathrm{a}+3$, sendo que o sinal negativo indica maior freqüência e o positivo menor freqüência de resposta. Assim, nas mulheres as respostas sempre ficaram no intervalo de -1 a -8 e nos homens no intervalo -1 a -7 . As respostas nunca, nas mulheres, ficaram compreendidas no intervalo +1 a +3 (menos freqüentes) e nos homens no intervalo $+1 \mathrm{a}+4$. 
Sisto, F. F., Bartholomeu, D., Santos, A. A. A., Rueda, F. J. M. \& Suehiro, A. C. B. (2008). Funcionamento Diferencial de Itens para Avaliar a Agressividade de Universitários.

Tabela 3

Medidas de DIF por Sexo, Mudança e Valores de t, por Item $(N=445)$ com Diferença Significativa

\begin{tabular}{|c|c|c|c|c|c|c|}
\hline Item & Sexo & DIF acrescido & Sexo & DIF acrescido & Mudança & $\mathrm{t}$ \\
\hline \multicolumn{7}{|c|}{ Itens mais freqüentes ao sexo masculino } \\
\hline A11 & Feminino & 0,96 & Masculino & $-0,50$ & 1,46 & 2,91 \\
\hline A12 & Feminino & 1,01 & Masculino & $-0,54$ & 1,54 & 3,88 \\
\hline A13 & Feminino & 0,71 & Masculino & $-0,56$ & 1,26 & 6,56 \\
\hline A17 & Feminino & 0,58 & Masculino & $-0,39$ & 0,97 & 2,42 \\
\hline A20 & Feminino & 0,41 & Masculino & $-0,39$ & 0,80 & 4,67 \\
\hline A29 & Feminino & 1,10 & Masculino & $-0,53$ & 1,63 & 2,56 \\
\hline A 34 & Feminino & 0,40 & Masculino & $-0,44$ & 0,84 & 5,71 \\
\hline A38 & Feminino & 0,45 & Masculino & $-0,71$ & 1,16 & 7,77 \\
\hline A39 & Feminino & 0,62 & Masculino & $-0,43$ & 1,05 & 3,58 \\
\hline A40 & Feminino & 2,09 & Masculino & $-0,89$ & 2,99 & 8,89 \\
\hline A47 & Feminino & 0,23 & Masculino & $-0,23$ & 0,47 & 2,44 \\
\hline A66 & Feminino & 0,92 & Masculino & $-0,58$ & 1,50 & 5,74 \\
\hline A67 & Feminino & 0,27 & Masculino & $-0,28$ & 0,55 & 3,33 \\
\hline A76 & Feminino & 2,14 & Masculino & $-0,74$ & 2,88 & 5,54 \\
\hline \multirow[t]{2}{*}{ A81 } & Feminino & 1,95 & Masculino & $-0,64$ & 2,59 & 2,49 \\
\hline & & & \multicolumn{4}{|c|}{ Itens mais freqüentes ao sexo feminino } \\
\hline A3 & Feminino & $-0,22$ & Masculino & 0,30 & $-0,53$ & $-3,78$ \\
\hline A10 & Feminino & $-0,30$ & Masculino & 0,42 & $-0,72$ & $-2,66$ \\
\hline A15 & Feminino & $-0,35$ & Masculino & 0,54 & $-0,89$ & $-3,64$ \\
\hline A 22 & Feminino & $-0,24$ & Masculino & 0,33 & $-0,58$ & $-3,00$ \\
\hline A24 & Feminino & $-0,24$ & Masculino & 0,33 & $-0,57$ & $-4,07$ \\
\hline A25 & Feminino & $-0,32$ & Masculino & 0,45 & $-0,77$ & $-5,42$ \\
\hline A41 & Feminino & $-0,17$ & Masculino & 0,23 & $-0,40$ & $-2,86$ \\
\hline A51 & Feminino & $-0,31$ & Masculino & 0,44 & $-0,75$ & $-4,65$ \\
\hline A56 & Feminino & $-0,33$ & Masculino & 0,47 & $-0,80$ & $-5,51$ \\
\hline A60 & Feminino & $-0,27$ & Masculino & 0,38 & $-0,66$ & $-4,67$ \\
\hline A61 & Feminino & $-0,42$ & Masculino & 0,69 & $-1,12$ & $-6,03$ \\
\hline A62 & Feminino & $-0,15$ & Masculino & 0,20 & $-0,34$ & $-2,43$ \\
\hline A69 & Feminino & $-0,33$ & Masculino & 0,46 & $-0,79$ & $-5,67$ \\
\hline
\end{tabular}

\begin{tabular}{|c|c|c|}
\hline \multicolumn{2}{|c|}{ Mulheres } & Homens \\
\hline & & \\
\hline
\end{tabular}

Figura 1. Curva Característica do item 3 


\section{Discussão}

Este estudo foi proposto com base no fato de que, na literatura, vários estudos demonstraram diferenças de sexo nas manifestações agressivas (Archer, 1991; Cairns et al., 1989; Graham et al., 2004; Sisto, 2003; Sisto et al., 2005). Assim, supôs-se que as condutas agressivas de itens elaborados com base nos critérios diagnósticos dos transtornos de conduta que são mencionados no DSM-IV (APA, 1995) e CID-10 (OMS, 1993), poderiam ser mais comuns em um ou outro sexo.

Nesse contexto, investigou-se o funcionamento diferencial dos itens $(D I F)$ de uma escala de agressividade, construída com base nos critérios diagnósticos anteriormente mencionados, por meio do modelo de análise sugerido por Rasch (1960), uma vez que ele possibilita calcular a probabilidade de acerto aos itens, por pessoa num determinado nível de habilidade, considerando também suas dificuldades. Esse tipo de informação é considerado como uma evidência de validade de construto, já que informa o grau em que cada item estaria mensurando um traço determinado ou construto, identificando quais deles estariam enviesados favorecendo um ou outro grupo (APA, AERA, \& NCME, 1999; Sisto, 2006; Sisto et al., 2005). Anteriormente à análise do $D I F$, esses itens foram submetidos a uma análise de conteúdo, sendo que os com conteúdos comuns ou com falta de concordância entre os examinadores foram excluídos e os que possuíam problemas de compreensão foram modificados.

A análise de $D I F$ evidenciou a existência de itens que favoreciam tanto o sexo masculino como o feminino. Os itens que favoreceram as mulheres, de forma geral indicaram uma tendência a se irritarem facilmente, a fazerem fofocas, a descontarem nos outros quando sentem raiva, a alterarem o tom da voz quando não são atendidas, entre outras manifestações. Em contrapartida, os itens que demonstraram maior favorecimento ao sexo masculino revelaram que os homens tendem mais a xingar pessoas, pôr apelidos maldosos nos outros, mentir, incomodar, violar regras, bater em placas, punir animais, entre outras condutas.

Considerou-se que os procedimentos de DIF seriam apropriados para captar o viés potencial de um item, já que não possibilitam uma explicação teórica para suas causas. Dessa forma, os resultados estatísticos obtidos deveriam ser interpretados à luz da teoria ou com o auxílio de peritos no assunto. Ressalta-se ainda que os vieses detectados podem ser parte legítima do construto, devendo-se examinar cautelosamente o conteúdo desses itens (Andriola, 2001; Sisto, 2006).

Nesses termos, e considerando que quase metade dos itens da escala apresentou possibilidade de viés, pode-se pensar que haveria manifestações agressivas diferentes em razão do sexo, o que é corroborado pela literatura já mencionada. Assim, existe a chance de haver fatores diferentes nessas condutas ao se considerar os sexos separadamente. Os resultados aqui obtidos são congruentes com os encontrados em pesquisas mais recentemente desenvolvidas (Carr \& VanDeusen, 2004; Fillmore \& Weafer, 2004; Graham et al., 2004).
No trabalho de Sisto et al. (2005), os autores sugeriram que os homens tenderam mais a importunar os demais, destruir algo que não lhes pertence, enganar, violar regras, desrespeitar a segurança própria e alheia, além de culpar os outros por coisas que fizeram, coagir pessoas e não se arrepender por atos cometidos, comportamentos também identificados na presente pesquisa, uma vez que os itens que favoreceram esse grupo envolvem condutas dessa natureza. Já as mulheres foram caracterizadas por Sisto, Bartholomeu, et al. (2005) como manipuladoras, irritadiças, com humor deprimido, baixa tolerância a frustrações além de fazerem birra para alcançar objetivos e brigarem freqüentemente. Esses resultados estão em consonância com os ora encontrados, embora os procedimentos para se obter os resultados tenham sido diferentes.

Archer (1991) demonstrou em seus estudos que as agressões físicas seriam mais características dos homens. No presente estudo, não houve alusões diretas para esse aspecto, não se podendo confirmar esse dado. Além disso, as mulheres demonstraram o uso de comportamentos agressivos indiretos ou não agressivos diretamente (como por exemplo, nos itens: "Durante uma conversa não deixo espaço para o outro se colocar", "Falo dos outros pelas costas", 'Informo a meus pais onde realmente vou quando saio", "Quando não gosto de alguém sinto vontade de esconder a verdade para prejudicá-lo", que favoreceram as mulheres), o que foi constatado por Cairns et al. (1989) em sua pesquisa.

Entretanto, MacIntyre e Cantrell (1995) assim como Wells e Graham (2003) e Fillmore e Weafer (2004) encontraram em seus trabalhos um maior nível de agressividade em homens em comparação às mulheres. O presente estudo não possibilitou observar esse aspecto, todavia, a investigação de Pulkkinen et al. (2000) pôde ser corroborada em partes já que, alguns dos indicadores encontrados que demonstraram caracterizar o sexo masculino foram justamente condutas anti-sociais, impulsividade e problemas de socialização. Além do que, as mulheres demonstraram também menos indicações de baixa sociabilidade, o que foi verificado por esses autores.

Desse modo, pode-se sugerir, reafirmando o que a literatura sobre o tema apresenta, que existem variações em relação ao sexo dos indivíduos no que tange às manifestações da agressividade, diferenças estas, já mencionadas anteriormente. Nesses termos, as avaliações dessa variável, devem ser realizadas tomando-se em conta tais diferenças, o que corrobora o ponto de vista de Sisto et al. (2005). Um aspecto que não foi observado na revisão de literatura feita foi que, embora se tenha identificado diferenças nessas manifestações, nenhum dos testes encontrados apresentou escalas separadas em razão dessa variável, o que pode introduzir um viés ao se interpretar os resultados fornecidos por essas medidas.

Ademais, novas pesquisas devem ser realizadas buscando outras evidências de validade para essa escala. Pode-se analisar a estrutura fatorial da mesma, levando-se em consideração as diferenças aqui apontadas. 
Sisto, F. F., Bartholomeu, D., Santos, A. A. A., Rueda, F. J. M. \& Suehiro, A. C. B. (2008). Funcionamento Diferencial de Itens para Avaliar a Agressividade de Universitários.

\section{Referências}

American Psychiatric Association. (1995). Manual diagnóstico e estatístico de transtornos mentais (4. ed.). Porto Alegre, RS: Artes Médicas.

American Psychological Association, American Educational Research Association, \& National Council on Measurement in Education (1999). Standards for educational and psychological testing. Washington, DC: American Psychological Association.

Andriola, W. B. (2001). Descrição dos principais métodos para detectar o funcionamento diferencial dos itens (DIF). Psicologia: Reflexão e Crítica, 14(3), 643-652.

Aquino, J. R. G. (1996). Confrontos na sala de aula - Uma leitura institucional da relação professor-aluno. São Paulo, SP: Summus.

Archer, J. (1991). The influence of testosterone on human aggression. Behavior-Genetics, 9(6), 571-577.

Bond, T. G., \& Fox, C. M. (2001). Applying the Rash Model: Fundamental measurement in the Human Sciences. London: Lawrence Erlbaum.

Cairns, R. B., Cairns, B. D., Neckerman, H. J., Ferguson, L. L., \& Gariepy, J. (1989). Growth and aggression: Childhood to early adolescence. Developmental Psychology, 25, 320-330.

Carr, J. L., \& VanDeusen, K. M. (2004). Risk factors for male sexual aggression on college campuses. Journal of Family Violence, 19(5), 279-291.

Draba, R. E. (1977). The identification and interpretation of item Bias (Research Memorandum No. 25). Chicago: The University of Chicago, Department of Education, Education Statistics Laboratory. Retrieved April, 2004, from http://www.rasch.org/ $\mathrm{rmt} / \mathrm{rmt} 122 \mathrm{~m} \cdot \mathrm{htm}$

Fillmore, M. T., \& Weafer, J. (2004). Alcohol impairment of behavior in men and women. Addiction, 99, 1237-1246.

Gomide, P. C. (2000). A influência de filmes violentos em comportamento agressivo de crianças e adolescentes. Psicologia Reflexão e Crítica, 13(1), 25-34.

Graham, K., Plant, M., \& Plant, M. (2004). Alcohol, gender and partner aggression: A general population study of British adults. Addiction Research and Theory, 4, 385-401.

Hambleton, R. K. (1989). Introduction. International Journal of Educational Research, 13, 123-125.

Huesmann, L. R., Eron, L. D., Ledfkowitz, M. M., \& Walder, L. O. (1984). The stability of aggression over time and generations. Developmental Psychology, 20, 1120-1134.

Jensen, A. R. (1980). Bias in mental testing. New York: The Free Press.

Jozef, F., Silva, J. A. R., Greenhalgh, S., Leite, M. E. D., \& Ferreira, V. H. (2000). Comportamento violento e disfunção cerebral: Estudo de homicidas no Rio de Janeiro. Revista Brasileira de Psiquiatria, 22(3), 124-129.

Kolko, D. J., Kazdin, A. E., \& Meyer, E. C. (1985). Aggression and psychopathology in childhood firesetters: Parent and child reports. Journal of Consulting and Clinical Psychology, 53(3), 377-385.

Linacre J. M. (2002). What do infit and outfit, mean-squared and standardized mean? Rasch Measurement Transactions, 16(2), 878, Retrieved June 10, 2006, from http://209.238.26.90/ $\mathrm{rmt} / \mathrm{rmt} 82 \mathrm{a} \cdot \mathrm{htm}$

MacIntyre, D. I., \& Cantrell, P. J. (1995). Punishment history and adult atittudes towards violence and aggression in men and women. Social Behavior and Personality, 23(1), 23-28.
Maldonado, D. P. A., \& L. C. A. Williams, (2005). O comportamento agressivo de crianças do sexo masculina na escola e sua relação com a violência doméstica. Psicologia em Estudo, 10(3), 353-362.

Meijer, M. D. (1991). The attribution of aggression and grief to body movements: The effect of sex-stereotypes. European Journal of Social Psychology, 21, 249-259.

Moreira, M. S. (1982). A violência dos grandes centros urbanos. Jornal Brasileiro de Psiquiatria, 3(31), 179-184.

Olweus, D. (1991). Bully-Victim problems among school children: Basic facts and effects of a school-based intervention program. In K. Rubin \& D. Pepler (Eds.), The development and treatment of childhood aggression (pp. 85-128). Hillsdale, NJ: Erlbaum.

Olweus, D. (1993). Bullying at school. Cambridge, MA: Blackwell.

Organização Mundial de Saúde. (Ed.). (1993). Classificação de transtornos mentais e de comportamento da CID-10. Descrições clínicas e diretrizes diagnósticas. Porto Alegre, RS: Artes Médicas.

Pulkkinen, L., Virtanen, T., Klinteberg, B. A., \& Magnusson, D. (2000). Child behaviour and adult personality: Comparisons between criminality groups in Finland and Sweden. Criminal Behaviour and Mental Health, 10, 155-169.

Rasch, G. (1960). Probabilistic models for some intelligence and attainment tests. Copenhagen, Denmark: Danmarks Paedogogiske Institut.

Sisto, F. F. (2003). Rejeição entre colegas e agressividade na infância. Cadernos de Psicologia, 13, 83-98.

Sisto, F. F. (2005). Aceitação-rejeição para estudar e agressividade na escola. Psicologia em Estudo, 10(1), 117-125.

Sisto, F. F. (2006). O funcionamento diferencial do item. Psico$U S F, 11,35-43$.

Sisto, F. F., Bartholomeu, D., Rueda, F. J. M., \& Granado, J. I. (2005). Condutas agressivas e gênero: Uma questão de estilo de agressividade. In M. C. R. A. Joly, A. A. A. Santos, \& F. F. Sisto, Questões do cotidiano universitário (pp. 141-158). São Paulo, SP: Casa do Psicólogo.

Sisto, F. F., \& Bazi, G. A. P. (2000). Escala de agressividade para crianças e jovens (Relatório Técnico). São Paulo, SP: Universidade Estadual de Campinas.

Sisto, F. F., Fernandes, D. C., \& Fernandes, D. C. (2006). Estudo do funcionamento diferencial dos itens do Teste de Raciocínio Inferencial. In C. Machado, L. Almeida, M. A. Guisande, M. Gonçalves, \& V. Ramalho, Avaliação psicológica: Formas e contextos (pp. 81-90). Braga, Portugal: Psiquilíbrios.

Sisto, F. F., Noronha, A. P. P., \& Santos, A. A. A. (2005). Bender - Sistema de pontuação gradual B-SPG. São Paulo, SP: Vetor.

Sisto, F. F., Rueda, F. J. M., \& Bartholomeu, D. (2006). Estudo sobre a unidimensionalidade do teste Matrizes Progressivas Coloridas de Raven. Psicologia: Reflexão e Crítica, 19(1), 66-73.

Wells, S., \& Graham, K. (2003). Aggression involving alcohol: Relationship to drinking patterns and social context. Addiction, 98, 33-42.
Recebido: 03/04/2007 $1^{a}$ revisão: $17 / 12 / 2007$ Aceite final: $10 / 05 / 2008$ 